\title{
Automatic Segmentation of Multiple Sclerosis Lesions in Brain MR Images
}

\author{
${ }^{1}$ Mohaddeseh Peyvandi and ${ }^{2}$ Ali A. Pouyan \\ School of Computer Engineering, Shahrood University of Technology, Shahrood, Iran; \\ 1peyvandisogand@gmail.com; ªpouyan@shahroodut.ac.ir
}

\begin{abstract}
Magnetic resonance (MR) imaging is one of the most important tools for multiple sclerosis (MS) diagnosis. However, detection and segmentation of MS lesion in MR images is challenging. Variability in lesion location, size, shape, and anatomical variability between subjects are some factors that cause accurate identification of MS lesions in MR images extremely difficult. On the other hand, manual segmentation is time-consuming. Furthermore, it is subject to observer variability. Several methods have been proposed to automatically segment MS lesions. They have been classified as supervised and unsupervised approaches. In this paper, we used both strategies, using combination of hidden Markov random field (HMRF), k-nearest neighbors (KNN) and support vector machine (SVM) algorithms. The performance of proposed approach is quantitatively evaluated on $20 \mathrm{MS}$ patients that have provided by MS lesion segmentation grand challenge dataset (MICCAI 2008). The average value of dice coefficient percentage (80.03\%) and Positive Predictive Value $(0.7661)$ are computed by spatially comparing the results of present procedure with expert manual segmentation. The results showed acceptable performance for the proposed approach, compared to those of previous work.
\end{abstract}

Keywords: Multiple Sclerosis; MS lesion; MR image; Brain segmentation.

\section{Introduction}

Multiple sclerosis (MS) is known as a chronic inflammatory disease of the central nervous system (CNS) [1]. In people with MS, immune system damage to the isolating layer of myelin around the nerve fibers in the brain, spinal cord and optic nerves that creates lesions and CNS atrophy in both grey matter (GM) and white matter (WM) tissue. With the progression of plaques, carries nerve impulses along the nerves become slow, which cause the symptoms of MS.

Brain imaging is a promising field for the diagnosis of MS that not used only to raise percentage of diagnosis, but also monitoring of disease progression and treatment efficacy. Since MS plaques are visible on brain tissue and anatomy, experts are used magnetic resonance (MR) images which show a good discrimination between tissues. Another characteristic of MR images is imaging of members of body in axial, coronal and sagittal directions. Conventional MR images such as T1-weighted (T1), T2-weighted (T2), proton density-weighted (PD) and fluid attenuated inversion recovery (FLAIR) are sensitive to lesions and show them with different intensity to peripheral tissues. Also intensity of MS lesions and brain tissues is different in various MR sequences [2]. Accordingly, the use of multispectral images is necessary to achieve 
an efficient algorithm. Lesions are often classified into three main groups based on their intensity under different MR sequences: T2 hyperintense lesions, gadolinium (Gd)-enhanced lesions and black holes [3].

Segmentation methods are mainly dependent on characteristics, application and quality of MR images. Imaging parameters, noise and artifacts, partial volume effects, non-uniform intensity, the patient movement during imaging and differences in the topological architectures of human brain, among the reasons for segmentation of MS lesions in MR images is difficult.

Survey papers in the field of automatically segmentation of MS lesions; have presented different categories of MS lesion segmentation approaches. Reference [1] suggested dividing the algorithms into two categories: supervised and unsupervised methods. Supervised group use prior knowledge such as atlas registration and manual segmentation (based on training) for the detection of MS lesions (i.e. Bayes classifiers with labeled maximum likelihood estimators, KNN and artificial neural networks (ANN)) and unsupervised group is divided into those techniques that use tissue segmentation to obtain the lesions and those that use only the lesion properties for the segmentation (i.e. Bayes classifiers with unlabeled maximum likelihood estimators or the fuzzy C-means (FCM) algorithms).

Another point of view, Classification methods can be grouped into two main approaches: class-based and outlier-based methods [4]. In the class-based methods, MS lesions are classified in a separate class along the same as three brain tissues [5]. In the outlier-based methods, brain tissues are assigned to three classes while MS lesions are delineated as outliers [6]. Since the number of voxels of the lesions class is much less than voxels belonging to other tissues, the estimated probability density function for the lesions class, is not as accurate as those of the other classes. As a result, it is expected that the outlier-based methods generate more accurate segmentation of the MS lesions.

In [4], some key individual classification methods are compared in terms of accuracy, time calculation, memory storage and computational complexity. Based on this comparison, there is a trade-off between accuracy and time calculation. Essentially, high precision spends more time. According to the evaluation proposed methods, in order to reach to an efficient and practical approach, it is necessary to consider a few points: the use of multispectral MR images, spatial features, manual segmentation and atlas registration. Moreover, segment lesion as outliers.

In order to consider above assumptions, it's necessary to present a unified approach of supervised and unsupervised methods. In the present study, we developed and implemented HMRF unsupervised method (that has been reported as the most successful method for segmentation of brain tissues [7]) to classify three main brain tissues on the high resolution 3D images. This method using of multi-channel images and without need expert segmentation, provides high-precision results in the field of brain tissues segmentation and since the inherently uses spatial features, the error due to noise and non-uniform intensity of tissues is reduced. We obtained initial segmentation of brain tissues thanks to a priori registration with the statistical atlas and without considering a separate class for MS lesions in the segmentation step, lesions are delineated as outlier at the post-processing step. On the other hand, we implemented SVM and KNN methods as training-based approaches to using real characteristics of the brain tissues and lesions. Finally, by combining the results of three segmentation methods achieved acceptable results. In this study, the focus is only on the classification of lesions have surrounded by WM (WML) from FLAIR sequences. We also validated the WML classification using quantitative analyses. 
Mohaddeseh Peyvandi and Ali A. Pouyan; Automatic Segmentation of Multiple Sclerosis Lesions in Brain MR Images. Journal of Biomedical Engineering and Medical Imaging, Volume 2, No 5, October (2015) , pp 21-34

\section{Material and Methods}

\subsection{Patients and MR images}

The datasets were obtained from the MS lesion segmentation grand challenge (Medical Image Computing and Computer Assisted Intervention, MICCAI 2008) that has provided MR brain images for validating MS lesions segmentation technique (http://www.ia.unc.edu/MSseg/). The MRI data (with different volumes of MS lesions) were acquired on a 3T Siemens MRI scanner by the Children's Hospital Boston (CHB) and the University of North Carolina (UNC). In total, the brain MRI volumes of $45 \mathrm{MS}$ patients were randomly divided into training sets ( 10 from each source), and testing sets ( 15 from $\mathrm{CHB}, 10$ from UNC). The training sets also include expert lesion segmentation. But the lesion segmentation on the testing data obtained by the CHB and the UNC experts are not available to the public. Therefore, we just exploited the training sets to evaluate the method.

For all cases, the datasets contained the same number of high resolution images: a T1-w, a T2-w and a FLAIR image that are co-registered and re-sampled to $512 \times 512 \times 512$ with voxel dimensions of $0.5 \times 0.5$ $\times 0.5 \mathrm{~mm} 3$. In this study, the segmentation method has been applied only to the 20 slices of MR images.

\subsection{Automated Segmentation of MS Lesions}

The six major components of the proposed segmentation technique are: (1) image pre-processing, (2) segmentation of brain tissues by HMRF (3) detection of lesions as outliers, (4) segmentation of WML by KNN, (5) segmentation of WML by SVM and (6) combining classifiers.

\subsection{Image pre-processing}

Image pre-processing will has a significant impact on the accuracy of the segmentation results. The image pre-processing includes co-registration, Image cropping, Skull-stripping, intensity non-uniformity correction, noise reduction and intensity normalization. It is important that the steps be performed respectively because it affects to improve image quality. MR sequences of MICCAI 2008 dataset are already co-registered. This means that a same voxel in the different sequences represents the same location in the brain.

In order to reduce the computational complexity, removed the number of voxels belonging to the background and decreased the image size to $390 \times 290$. The majority of Skull-stripping techniques extract the intracranial space from the image. For this purpose, we have applied automated brain extraction algorithm to the T2 images by exploiting the brain extraction tools (BET) in the MRIcro software [8]. Following that skull stripped T2 image was used to generate the mask which was applied to the T1 and FLAIR images.

Difference in intensity of same biological tissues that generated by the bias field is called image inhomogeneities. In our case, we estimated and corrected these in-homogeneities with the N3 method proposed in [9]. Also, to reduce noise without blurring small morphological details an anisotropic diffusion filter was applied [10] and finally the intensity profiles of the images were normalized (range from 0 to 255) using the decile based piece-wise linear transformation technique [11]. 


\subsection{Segmentation of Brain Tissues by HMRF}

Hidden Markov random fields have been suggested in image processing in order to model neighborhood and spatial interactions. Methods relying on MRF define the image as a set of $\mathrm{N}$ discrete sites ( $\mathrm{N}$ is number of voxels) in which each site represents a voxel connected to its neighboring. Following Morkovian properties, assigning a label to each site only depends on label of its neighborhood set. In this study, we used HMRF algorithm to segment brain tissues. The FLAIR sequence provides a better distinction between lesions and normal tissue but the boundary between tissues has low resolution. Accordingly, tissues have been segmented only base on the features extracted from the T1 and T2 sequences [7].

Elimination of partial volume artifacts is one of the complexities of the MR images segmentation. In this case, a voxel may be composed of more than one tissue type. Partial volume problems arising from low resolution images or surface area is intended for a voxel that can be influence on the statistical properties such as mean and variance. To address this problem we have considered a separate class for voxels that are in the border areas between the two tissues [12]. In this approach, we defined two main types of classes: pure tissues which are assumed to follow a Gaussian mixture distribution and included WM, GM and cerebrospinal fluid (CSF) classes and partial volume classes which are defined as proportion between GM and CSF as well as WM and GM. While other interactions are excluded due to their slight influence.

MR images segmentation is performed in the following hierarchical procedure: (1) the probability of each voxel to belonging to the different classes is first initialized thanks to the a priori registration with the MNI atlas. At this stage, first a global affine transformation between the patient's T1 image and the atlas's template is estimated using mutual information and following that an initial segmentation of brain tissues is obtained using the module in SPM8 [13]; (2) The initial parameters of each class (mean and variance) are computed from the voxel intensities. Noting that the parameters of the partial volume classes are computed as a proportion of the pure tissue parameters, while the parameters of the background class are obtained as a fraction of the CSF parameters [12]; (3) Using current parameter set the labels of each voxel estimated by MAP estimation. This means that the label assigned to each voxel is updated based on label of its neighboring voxels [14]; (4) Based on labeled image obtained from the previous step, a new parameter set is estimated for each class using EM algorithm; (5) Steps 3 and 4 are repeated until a termination condition is achieved.

\subsection{Detection of MS Lesions as Outliers}

At the tissues segmentation stage, a class for lesions was not assigned. Therefore, Lesions incorrectly segmented in another classes due to intensity similarity with normal tissues. In this case, MS lesions are detected as outliers that do not follow the intensity Gaussian model. That means, distance between the intensity vector of outlier voxels and the mean vector of each class is greater than a threshold.

Lesions appear as hyperintense signals in FLAIR images. Moreover, GM voxels have the highest intensity in comparison with the other normal tissues in these images but its intensity is lower than the MS lesions. As a result, a significant difference between normal tissues and MS lesions appears in FLAIR images. Therefore lesion should be segmented as a GM-based class (pure GM or partial volume) or WM (black holes) that should always surrounded by WM tissue.

The aim of this step is apply a threshold on FLAIR images to find the candidate regions for lesions Class. First we assume GM intensities follow the Gaussian distribution. Also we assume that lesions are 
Mohaddeseh Peyvandi and Ali A. Pouyan; Automatic Segmentation of Multiple Sclerosis Lesions in Brain MR Images. Journal of Biomedical Engineering and Medical Imaging, Volume 2, No 5, October (2015) , pp 21-34

hyperintense outliers of GM class. According to these assumptions, a threshold on FLAIR image is defined as follows [15]:

$$
T=\mu_{G M}+\alpha \sigma_{G M}
$$

That $\mu_{\mathrm{GM}}$ and $\sigma_{\mathrm{GM}}$ are respectively the mean and standard deviation of GM on FLAIR images and $\alpha$ is an experimental parameter used to determine the outliers and set to 2 [16]. To estimate tissue parameters, we first applied GM mask that obtained in HMRF segmentation to FLAIR image and then calculated them by using the proposed method at the [17].

The application of this threshold on the FLAIR images help us to detect lesions have at least a voxel with intensity higher than the threshold. But due to intensity non-uniformity of lesion voxels, we enhanced the contrast in the FLAIR image thanks to morphological operations before plying the threshold [16].

After thresholding, we obtained an initial lesion mask while other hyperintense artifacts are included (false positive). In other hand the MS lesions include voxels outside the mask (false negative). Therefore, this mask must be refined, based on appearance and spatial characteristics of the MS lesions. For this purpose, we defined a set of rules that are true for WML as follow [15]:

- Lesions are mostly classified in WM and GM-based classes ( pure GM and partial )

- Lesions have surrounded by WM

- Lesions should not be present between ventricles

- Lesions should be of a minimum size.

- By applying these rules on the FLAIR images, the remaining regions will be introduced as MS lesions.

\subsection{Segmentation of WML by KNN}

In this section, we proposed a supervised segmentation method based on KNN classification that has been introduced in [18]. In this approach, a new voxel image is classified by the majority class of the K learning samples $(K=50)$ that are closest in terms of Euclidian distance to it. However, instead of assign one class to the voxel, determine the probability being a lesion per voxel by taking the number of lesion voxels among the $\mathrm{k}$ neighbors. As a result, a lesion probability map is constructed for every test image. Subsequently the map is threshold to derive a binary segmentation of the lesions. We defined an optimal threshold of 0.4 to apply on all probabilistic segmentations of the testing sets.

\subsection{Segmentation of WML by SVM}

SVM classifier has provided satisfactory results as a binary classification task like the one in our study (normal tissues/MS lesion) [19]. Initially a set of training samples that manually segmented by expert used to build a classification model via SVM. Then the SVM model is used to perform the voxel-wise segmentation. This means, lesion rating for each voxel (likelihood of being lesion) is estimated by the generated SVM classifier where each voxel is assigned with values in the range $[-1,1]$. If this value is close to -1 , probabilities that a voxel belongs to tissues class is more than lesion class and if it close to 1 more likely to belong to the lesion class. Finally the output of SVM is binarized by an optimal threshold of 0.8 to produce the labels for MS lesions.

It should be noted, because of the large number of voxels in the training sets only $30 \%$ of lesion voxels and $5 \%$ of tissues voxels are randomly selected as the training points. Also the feature space have been 
used in supervised methods (KNN and SVM) are included voxel intensity of FLAIR Images and voxel location in the brain that is uniquely defined by the $x, y$ and $z$ coordinates. These features further are normalized to have zero mean and variance of one.

\subsection{Combining Classifiers}

Numerous experiments have been conducted on combining classifiers [20]. The main objective of designing different classification schemes is to achieve the best classification. Considering the situations (different classifiers with different features) we developed Majority Vote rule to combine results of three classifiers [21]. The Maximum rule counts the votes for each class over the input classifiers and selects the majority class. In general, at each value of $X$ a majority vote classifier consisting of votes from rules $\mathrm{h}_{1}, \mathrm{~h}_{2}, \ldots, \mathrm{h}_{\mathrm{b}}$ is defined as follows:

$$
C(X)=\arg \max _{i} \sum_{j=1}^{B} w_{j} I\left(h_{j}(X)=i\right)
$$

Where $\mathrm{w}_{1}, \mathrm{w}_{2}, \ldots, \mathrm{w}_{\mathrm{b}}$ are weights of classifiers $1,2, \ldots, \mathrm{B}$ that sum to 1 and $\mathrm{I}$ is an indicator function is define as follows:

$$
I(y)=\left\{\begin{array}{lll}
1: & \text { if } y=\text { true } \\
0: & \text { if } y=\text { false }
\end{array}\right.
$$

We assume equal weights for all classifiers in which case all the weights $w_{i}$ set to 1/B.

\section{Validation}

We have used K-fold cross-validation method to evaluate performance of proposed method. In this approach, the dataset equally is divided into $k$ subsets. Then for each of $k$ experiments, $k$ - 1 folds for training and the remaining one for testing are used. So that the all samples in the dataset are eventually used for both training and testing. Finally, the accuracy of proposed method is obtained as the average of $k$ separate estimates. In our problem, we have $k=4$ subsets.

We have implemented the segmentation method in Matlab-code using a dual core processor $2.1 \mathrm{GHz}$ and 3 GB RAM. Specifically, we used libSVM in Matlab to develop SVM classifier [22]. The kernel function has been used in our application is Gaussian radial basis function (RBF).

\subsection{Evaluation measures}

Various measures are used to evaluate the proposed methods for the segmentation of the MS lesions. The Lesions have detected by the expert were considered as the reference lesion classification (ground truth). The automatic segmentation results and ground truth can be compared by either comparing each voxel in each lesion (voxel to voxel), or using the total lesion load (lesion-to-lesion). In both cases, twoclass classification model for image segmentation is implemented in which voxels and lesions can be classified as a true positive (TP), false positive (FP), true negative (TN) or false negative (FN). In our problem, we assigned a class to lesions and a class to other regions of image (including brain tissues and background voxels).

The quantitative metrics used for the evaluation included dice similarity coefficient (DSC), absolute volume difference percent (Vol_Diff) and average symmetric surface distance (Avg_Dist) defined as: 


$$
\mathrm{DSC}=\frac{2 \times \mathrm{TP}}{2 \times \mathrm{TP}+\mathrm{FP}+\mathrm{FN}}
$$

Vol_Diff $=($ abs $($ reference volume - segmentation volume $) / * 100$

$$
\operatorname{Avg} \_ \text {Dist }=\left(\sum_{\mathrm{x} \in \partial(\operatorname{Ref})} \min _{\mathrm{y} \in \partial(\operatorname{Seg})} \mathrm{d}(\mathrm{x}, \mathrm{y})+\sum_{\mathrm{x} \in \partial(\operatorname{Seg})} \min _{\mathrm{y} \in \partial(\operatorname{Ref})} \mathrm{d}(\mathrm{x}, \mathrm{y})\right) /(\operatorname{card}(\operatorname{Seg})+\operatorname{card}(\operatorname{Ref}))
$$

Here, $\partial($ Ref) and $\partial(\mathrm{Seg})$ represent sets of border voxels of the ground truth (Ref) and segmented lesions (Seg) and the term card(Ref) and card(Seg) denote the number of border lesion voxels. For each voxel $(x)$ along one border, the closest voxel $(y)$ along the other border in term of Euclidean distance $(d(x, y))$ is determined. The average of all these minimum distances for border voxels from both reference and segmentation gives the average symmetric surface distance. Vol_Diff and Avg_Dist for a perfect segmentation should be close to 0 and DSC conformity index should be close to 1 . Practically, a DSC value greater than 0.7 indicates a good segmentation performance.

In addition, the ideal evaluation of the MS lesions segmentation methods should be based on multi-raters (the variable ground truth) using techniques such as the simultaneous truth and performance level estimation (STAPLE) [23]. For evaluation of the estimated lesions compared with the combined STAPLE segmentation the specificity, sensitivity, and positive predictive value (PPV) were calculated. PPV value provides a good measure combining both sensitivity and specificity. A good segmentation system should have high sensitivity and specificity values.

\subsection{Results}

The HMRF method classified the brain tissues into six different classes (Figure 1). The aim of this procedure is to obtain tissue masks that are used in the lesions segmentation step.

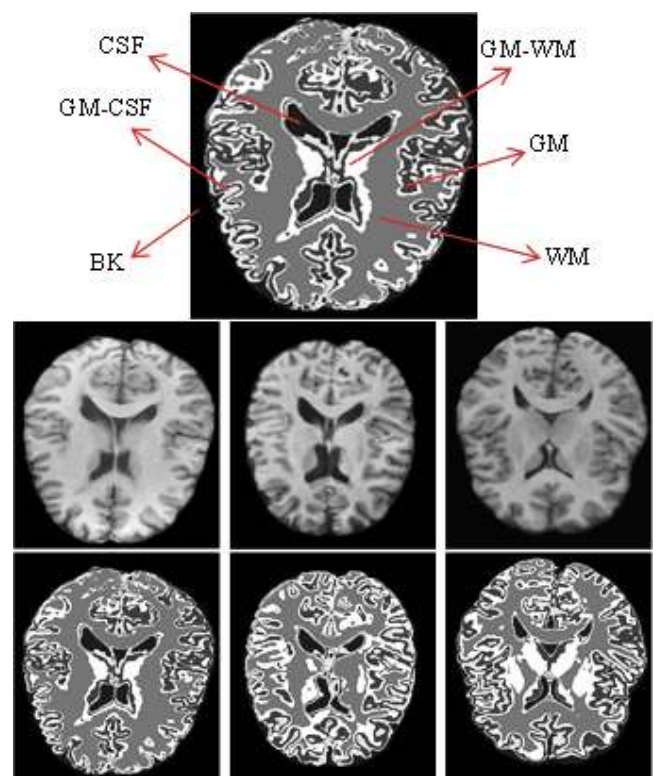

Figure 1: Tissue segmentation using HMRF: Top row: T1 images of some cases, bottom row: segmented images. 
In Figure 2 an example of the detected lesions; as outliers in the FLAIR images, have been shown. On the other hand, SVM and KNN classifiers in a two-class problem have been applied to detect MS lesions from the FLAIR images.

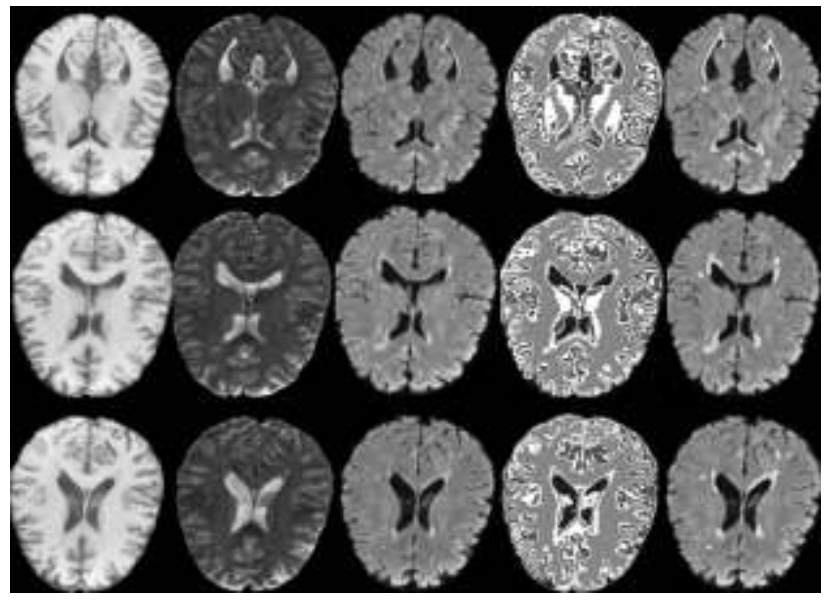

Figure 2: HMRF classification at various cross-sections of the CHB_Case02. First column: T1; second column:

T2; third column: FLAIR; fourth column: segmented tissues; and fifth column: segmented lesions.

Figure 3 shows the results of lesion segmentation based on supervised approaches. Finally, the obtained segmentations of the three classifiers are combined to achieve accurate results.
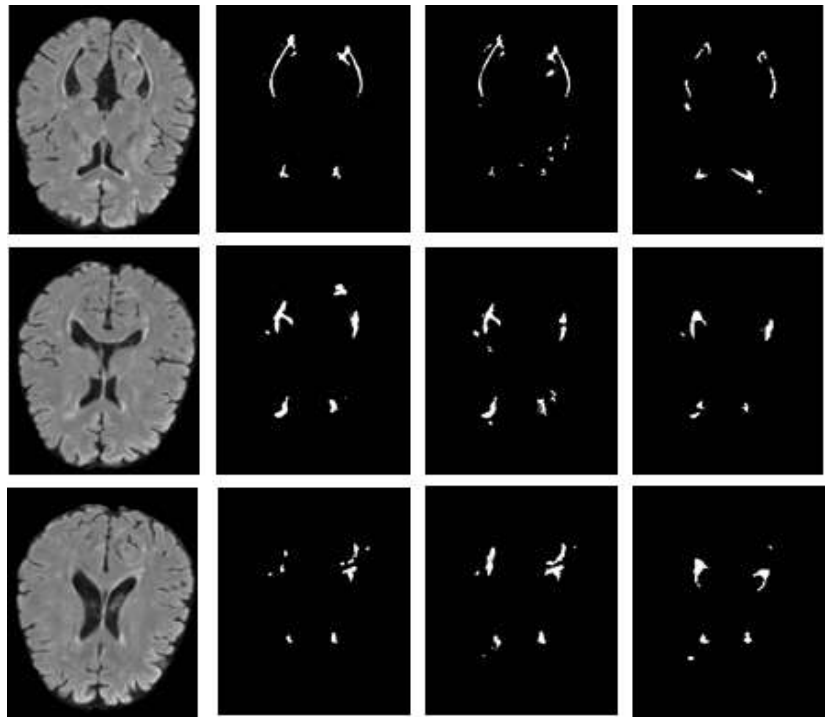

Figure 3: Supervised classification at various cross-sections of the CHB_Case02. First column: FLAIR image; second column: lesions segmentation by KNN classifier; third column: lesions segmentation by SVM classifier; fourth column: expert segmentation.

The final results of proposed methods are demonstrated in Figure 4. 


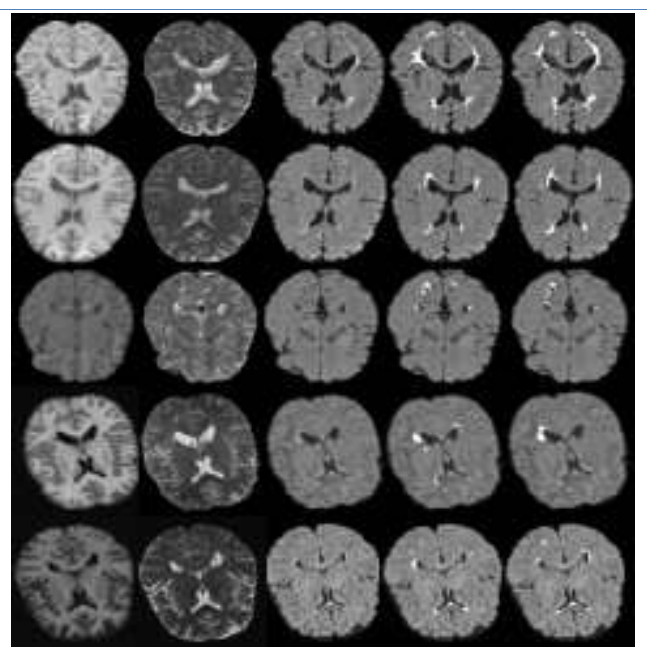

Figure 4: Automated segmentation of MS lesions at five cases from the MICCAI dataset. First column: T1; second column: T2; third column: FLAIR; fourth column: expert segmentation; and fifth column: segmented lesions by proposed method.

In Some cases with low quality of FLAIR images, the points with high signal intensity are observed to scattered noise within WM (e.g. UNC-case05, UNC-case09). These points due to having the same intensity with MS lesions are misclassified as lesions. Since, lesion segmentation step in HMRF approach only performed base on the intensity images, segmentation results of this approach provide less accurate than the supervised methods for this cases. Also, in reference images of some cases, experts have determined the voxels as MS lesions, which not included the characteristics of the two types of T2 hyperintense and black hole lesions (such as CHB-case01 and CHB-case10). In fact, the voxels have similar intensity with WM in FLAIR and T1 images. Therefore, it is expected that the detected lesions have been Gd-enhanced lesions. These lesions show an increase in intensity compared to WM on T1 images after injection with gadolinium. Since the dataset is not included the Gd-enhanced T1 images, the detection of this type of lesions is difficult.

Unlike HMRF approach in which the regions between the ventricles are firstly detected as MS lesions (and are removed of lesions class at the post-processing step), the supervised methods typically not segmented these regions in lesions class thanks to use location information of lesions. But as an advantage of HMRF, the details of image segmented by HMRF are much more than other segmentation methods. As a result, HMRF shows a better performance of the diagnosis of scattered MS lesions. Tissues segmentation also helps us to obtain the mask of brain tissues and by applying those on the MR images achieved three objectives: detecting the voxels of the tissues class that have similar intensity with MS lesions, defining region of interest for identify lesions occurring in WM and segmenting tissues with respect to the effects of partial volume.

It can be concluded that by understanding the advantages and limitations of the methods, can be represented a combining approach to achieve more accurate in the diagnosis of MS lesions. Note that cannot be represented definite results of the performance of the segmentation techniques that be true for all type of MR images and MS lesions. Because the characterizes have been defined for types of MS lesions may not be true in some regions of MR images, but an expert based on personal experiences has recognized these regions as MS lesions 


\subsection{Quantitative Evaluation}

The results of comparison between the automated segmentation and the ground truth are presented in Table 1.

Table 1. Results obtained on the public dataset of MICCAI.

\begin{tabular}{|c|c|c|c|c|c|c|c|c|c|c|c|c|}
\hline & \multicolumn{3}{|c|}{ HMRF } & \multicolumn{3}{|c|}{ KNN } & \multicolumn{3}{|c|}{ SVM } & \multicolumn{3}{|c|}{ Merge results } \\
\hline & 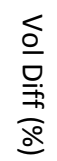 & 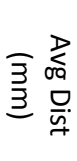 & $\frac{0}{\stackrel{n}{\circ}}$ & 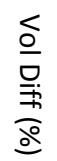 & 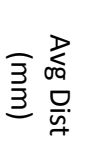 & $\frac{\tilde{n}}{\stackrel{0}{\circ}}$ & $\begin{array}{l}\text { o } \\
\underline{D} \\
\stackrel{0}{\circ}\end{array}$ & 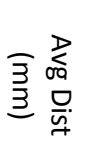 & $\frac{\stackrel{0}{n}}{\stackrel{0}{0}}$ & 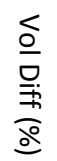 & 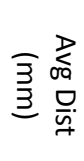 & 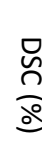 \\
\hline $\begin{array}{l}\text { Average all } \\
\text { dataset }\end{array}$ & $\underset{\vec{b}}{\vec{b}}$ & $\begin{array}{l}v \\
\dot{o} \\
\sim\end{array}$ & $\begin{array}{l}\text { ने } \\
\text { in }\end{array}$ & $\stackrel{w}{\mathscr{L}}_{\mathscr{\sigma}}$ & $\stackrel{\sim}{\Delta}$ & $\underset{\dot{\omega}}{\vec{\omega}}$ & $\begin{array}{l}\underset{n}{w} \\
\infty\end{array}$ & in & $\begin{array}{l}\grave{o} \\
\dot{\omega}\end{array}$ & $\stackrel{w}{w}$ & $i_{\omega}^{v}$ & $\begin{array}{l}\infty \\
\stackrel{0}{0} \\
\dot{\omega}\end{array}$ \\
\hline
\end{tabular}

Table 1 provides detailed quantitative metrics, DSC, Vol_Diff and Avg_Dist for the three classifiers and combined model. In order to facilitate the comparison, a bar chart of Dice coefficient for the three classifiers is shown in Figure 5.

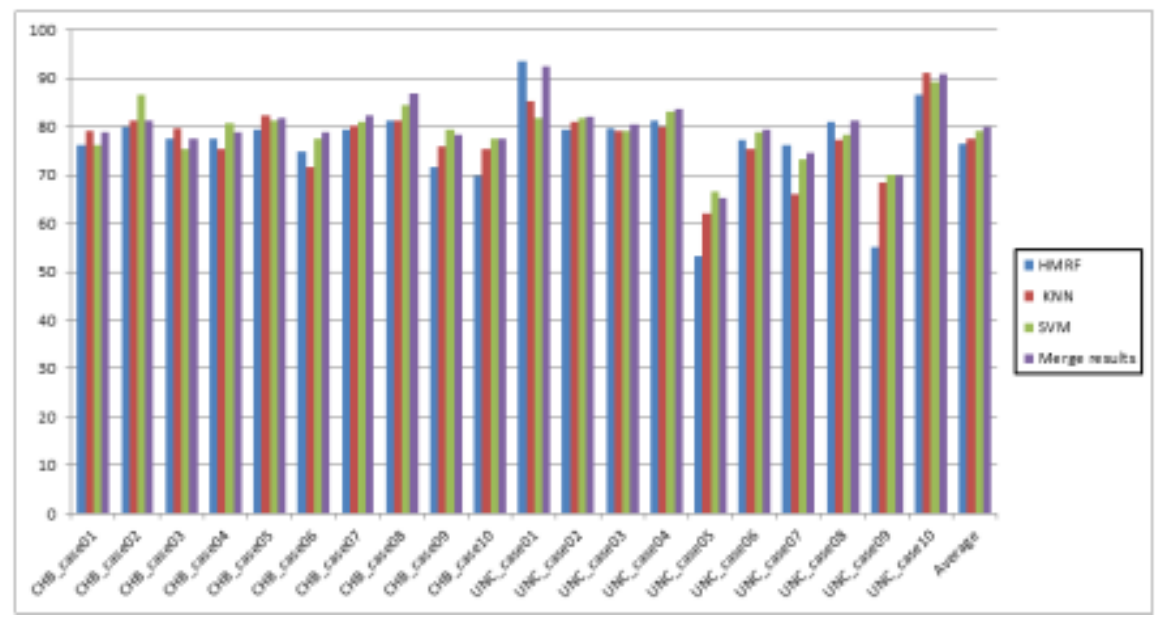

Figure 5: Bar chart for Dice coefficient.

Our technique received a DSC average value of 76.5, 77.43 and 79.13 percent for HMRF, KNN and SVM, respectively. We also calculated the DSC value for combined model. This improved the DSC value to 80.03 percent. The results indicate that the accuracy of MS lesions segmentation can be increased by combining the segmentation results of the three classifiers.

Note the cases with significant different lesion load can bias the results. The results of volumetric comparison of lesions between the automated segmentation and the ground truth are presented in Table 2.

Table 2. DSC values in MR images with different volumes of lesions.

\begin{tabular}{|c|c|c|}
\hline Patient category & Number of cases & DSC (\%) \\
\hline Small lesion load & 6 & 77.78 \\
\hline Moderate lesion load & 9 & 80.69 \\
\hline Large lesion load & 5 & 81.73 \\
\hline
\end{tabular}


Mohaddeseh Peyvandi and Ali A. Pouyan; Automatic Segmentation of Multiple Sclerosis Lesions in Brain MR Images. Journal of Biomedical Engineering and Medical Imaging, Volume 2, No 5, October (2015) , pp 21-34

For the patients with large lesion load, we reached to average value 81.73 for DSC which is more than those patients with small and moderate lesion load.

In Table 3 the segmentation results of our method were also compared with combined STAPLE segmentation. All binary segmentations of the 10 UNC images have been compared with manual segmentations of the two raters: a UNC rater and a CHB rater.

Table 3. Results of the segmentation method by STAPLE.

\begin{tabular}{|c|c|c|c|}
\hline \multirow{2}{*}{ UNC dataset } & \multicolumn{3}{|c|}{ STAPLE } \\
\cline { 2 - 4 } & Specificity & Sensitivity & PPV \\
\hline UNC_case01 & 0.999 & 0.6092 & 0.9545 \\
\hline UNC_case02 & 0.9972 & 0.5912 & 0.8741 \\
\hline UNC_case03 & 0.9995 & 0.4096 & 0.9448 \\
\hline UNC_case04 & 0.9995 & 0.4638 & 0.9015 \\
\hline UNC_case05 & 0.9871 & 0.1745 & 0.3175 \\
\hline UNC_case06 & 0.9937 & 0.4972 & 0.8283 \\
\hline UNC_case07 & 0.9953 & 1 & 0.4853 \\
\hline UNC_case08 & 0.9968 & 0.7734 & 0.8537 \\
\hline UNC_case09 & 0.9879 & 0.8977 & 0.6037 \\
\hline UNC_case10 & 0.9987 & 0.5999 & 0.8975 \\
\hline Average & 0.9955 & 0.6016 & 0.7661 \\
\hline
\end{tabular}

The average execution time in seconds was computed for all cases in dataset. According to Table 4, the total computational time for complete segmentation, including the preprocessing, feature extraction and lesions detection is 44.25 min that took considerable time. This time can be reduced using algorithms based on parallel computation.

Table 4. Average execution time of the method for 20 slices of MR images.

\begin{tabular}{|c|c|}
\hline Steps & Time (second) \\
\hline Pre-processing & 355 \\
\hline Feature extraction & 68 \\
\hline HMRF tissue segmentation & 1470 \\
\hline Lesion detection by tissue mask & 10 \\
\hline KNN classification & 672 \\
\hline SVM classification & 72 \\
\hline Merge results & 8 \\
\hline Total & $2655(44.25 \mathrm{~min})$ \\
\hline
\end{tabular}

Quantitative comparison among the approaches is a difficult task due to the variability in the datasets and evaluation measures. Table 5 provides a qualitative comparison of the DSC Coefficient obtained by the MS lesions segmentation approaches.

Table 5. DSC values for the proposed method and the other methods.

\begin{tabular}{|c|c|c|c|}
\hline Segmentation method & MR images & Number of samples & DSC (\%) \\
\hline EM+MRF [6] & T1, T2, PD & 50 & 51 \\
\hline ANN [24] & T1, T2, PD & 29 & 60 \\
\hline Parzen windows [25] & T2, PD, FLAIR & 23 & 78 \\
\hline Bayes+AMM+MRF [26] & FLAIR & 20 & 75.04 \\
\hline Simulated annealing +MRF [5] & T1, T2, PD & 10 & 71 \\
\hline FCM [27] & T1, T2, FLAIR & 10 & 80.03 \\
\hline Proposed method & T1, T2, FLAIR & 20 & \\
\hline
\end{tabular}


We have also provided a comparison among the approaches that have used of MICCAI dataset in Table 6.

Table 6: Comparison of the results of MS lesions segmentation on the MICCAI dataset.

\begin{tabular}{|c|c|c|c|c|c|}
\hline Segmentation method & Vol Diff (\%) & Avg Dist $(\mathbf{m m})$ & Specificity & Sensitivity & PPV \\
\hline KNN [18] & 46.9 & 7.8 & 0.9970 & 0.4519 & 0.9143 \\
\hline EM+GMM [16] & 86.5 & 8.2 & 0.9895 & 0.3583 & 0.6004 \\
\hline AdaBoost [28] & 133.4 & 13.2 & 0.9879 & 0.2893 & 0.5093 \\
\hline Bayes [29] & 174.6 & 22.2 & 0.9476 & 0.2984 & 0.4221 \\
\hline Proposed method & 35.7 & 7.93 & 0.9955 & 0.6016 & 0.7661 \\
\hline
\end{tabular}

Note that even a few cases with low quality images, have a significant negative effect on the average results of the proposed method.UNC-case09 and UNC-case05 with low quality of FLAIR images have decreased the average PPV value of the proposed methods.

\section{Conclusion}

We have presented and implemented a unified and automated approach for classifying MR brain images in MS. The aim of this unified approach is to combine the segmentation results from supervised and unsupervised methods for creating effective MS lesions segmentation. The proposed automated segmentation technique was assessed quantitatively for WML classification with the use of quantitative metrics on MICCAI dataset. The results of the automatic lesion segmentation were compared with lesion delineations by experts, showing a high total lesion load correlation. In the future, we will focus on studying the atrophy of brain and spinal cord, which is an important part of MS pathology.

\section{REFERENCES}

[1]. Lladó, X., et al., Segmentation of multiple sclerosis lesions in brain MRI: A review of automated approaches. Information Sciences, 2012. 186(1): p. 164-185.

[2]. Hashemi, RH., WG. Bradley and CJ. Lisanti, MRI: the basics. 3td edition, Philadelphia: Lippincott Williams and Wilkins, 2004. 400.

[3]. García-Lorenzo, D., et al., Review of automatic segmentation methods of multiple sclerosis white matter lesions on conventional magnetic resonance imaging. Medical Image Analysis, 2013. 17(1): p. 1-18.

[4]. Mortazavi, D., A. Z. Kouzani and, H. Soltanian-Zadeh, Segmentation of multiple sclerosis lesions in MR images: a review. Neuroradiology, 2012. 54(4): p. 299-320.

[5]. Subbanna, N., et al., MS lesion segmentation using Markov Random Fields. Medical Image Analasis Multiple Sclerosis, 2009: p. 15-26.

[6]. Leemput, KV., et al., Automated segmentation of multiple sclerosis lesions by model outlier detection. Medical Imaging, IEEE Transactions on, 2001. 20(8): p. 677-688. 
Mohaddeseh Peyvandi and Ali A. Pouyan; Automatic Segmentation of Multiple Sclerosis Lesions in Brain MR Images. Journal of Biomedical Engineering and Medical Imaging, Volume 2, No 5, October (2015) , pp 21-34

[7]. Zhang, Y., M. Brady and, S. Smith, Segmentation of brain MR images through a hidden Markov random field model and the expectation-maximization algorithm. Medical Imaging, IEEE Transactions on, 2001. 20(1): p. 45-57.

[8]. Smith, SM., Fast robust automated brain extraction. Human Brain Map, 2002. 17(3): p. 143-155.

[9]. Led, JG., AP. Zijdenbos and, AC. Evans, A nonparametric method for automatic correction of intensity nonuniformity in MRI data. Medical Imaging, IEEE Transactions on, 1998. 18(1): p. 8797.

[10]. Perona, P. and, J. Malik, Scale-space edge detection using anisotropic diffusion. Pattern Analysis and Machine Intelligence, IEEE Transactions on, 1990. 12(7): p. 629-639.

[11]. Nyúl, LG., GK. Udupa and, X. Zhang, New variants of a method of MRI scale standardization. Medical Imaging, IEEE Transactions on, 2000. 19(2): p. 143-150.

[12]. Tohka, J., A. Zijdenbos and, A. Evans, Fast and robust parameter estimation for statistical partial volume models in brain MRI. Neurolmage, 2004. 23(1): p. $84-97$.

[13]. Ashburner, J., et al., SPM8 Manual. 2013.

[14]. Patts-Shattuck, DW., et al., Magnetic resonance image tissue classification using a partial volume model. Neurolmage, 2001. 13(5): p. $856-876$.

[15]. Cabezas Grebol, M., PhD. Thesis, Atlas-based segmentation of multiple sclerosis lesions in magnetic resonance imaging. de Girona University, Spain, 2013.

[16]. Souplet, J., et al., An automatic segmentation of T2-FLAIR multiple sclerosis lesions. Multiple Sclerosis Lesion Segmentation Challenge 2008: p. 1-11.

[17]. de Boer, R., et al., White matter lesion extension to automatic brain tissue segmentation on MRI. Neuroimage, 2009. 45(4): p. 1151-61.

[18]. Anbeek, P., K. Vincken and, K. Viergever, Automated MS-lesion segmentation by k-nearest neighbor classification. Multiple Sclerosis Lesion Segmentation Challenge 2008: p. 1-8.

[19]. Laoa, Z., et al., Computer-assisted segmentation of white matter lesions in 3D MR images, using Support Vector Machine. Acad Radiol, 2009. 15(3): p. 300-313.

[20]. Duin, RPW. and DMJ. Tax, Experiments with classifier combining rules. Lecture Notes in Computer Science, 2000: p. 16-29.

[21]. James, G., PhD. Thesis, Majority vote classifiers: theory and applications. Stanford University, 1988.

[22]. Chang, C. and, C. Lin, LIBSVM: a library for support vector machines. 2001. 
[23]. Warfield, SK., KH. Zou and, WM. Wells, Simultaneous truth and performance level estimation (staple): an algorithm for the validation of image segmentation. Medical Imaging, IEEE Transactions on, 2004. 23(7): p. 903-921.

[24]. Zijdenbos, AP., R. Forghani and, AC. Evans. Automatic "pipeline" analysis of 3-D MRI data for clinical trials: Application to multiple sclerosis. Medical Imaging, IEEE Transactions on, 2002. 21(10): p. 1280-1291.

[25]. Sajja BR., et al., Unified approach for multiple sclerosis lesion segmentation on brain MRI. Annals of Biomedical Engineering, 2006. 34(1): p. 142-151.

[26]. Khayati, R., et al., Fully automatic segmentation of multiple sclerosis lesions in brain MR FLAIR images using adaptive mixtures method and Markov random field model. Comput Biol Med, 2008. 38(3): p. 379-390.

[27]. Shiee, N., et al., A topology-preserving approach to the segmentation of brain images with multiple sclerosis lesions. Neurolmage, 2010. 49(2) : p. 1524-1535.

[28]. Morra, J., et al., Automatic segmentation of MS lesions using a contextual model for the MICCAI grand challenge. Multiple Sclerosis Lesion Segmentation Challenge 2008: p. 1-7.

[29]. Scully, M., et al., 3D segmentation in the clinic: a grand challenge II at MICCAI $2008-M S$ lesion segmentation. Multiple Sclerosis Lesion Segmentation Challenge 2008: p. 1-9. 